BNL -48559

DE93 010864

\title{
ATF Beamline 1 Analysis Spectrometer
}

R. C. Fernow, H. G. Kirk and S. Ulc

Physics Department

Brookhaven National Laboratory

Associated Universities, Inc.

Upton, Long Island, New York 11973

Under Contract No. DE-AC02-76CH00016 with the UNITED STATES DEPARTMENT OF ENERGY 


\title{
ATF Beamline 1 \\ Analysis Spectrometer
}

\author{
R.C. Fernow, H.G. Kirk \& S. U1C
}

January 1993

\begin{abstract}
We describe the design parameters and expected performance of the analysis spectrometer for beamline 1 at the BNL Accelerator Test Facility. The spectrometer should be well suited for measuring the change in energy caused by the first generation laser acceleration experiments.
\end{abstract}

\section{Overview}

The ATF beamline 1 analysis spectrometer is intended primarily for measuring the energy gain in the advanced acceleration experiments. The Inverse-Cerenkov Acceleration[1] and Grating Acceleration[2] experiments, currently approved for running on this beamline, will use the spectrometer for this purpose. In addition other experiments, like the Non1inear-Compton scattering experiment[3], can use the spectrometer as a monitor of the electron beam-laser interaction.

The basic layout of the spectrometer is shown in Fig. 1. The beam leaving an interaction region encounters a vertically bending dipole, which is used to disperse the beam transversely as a function of momentum. Three quadrupoles, located in the space between the dipole exit face and the detector, are used to control the focal spot at the detector[4]. The detector is a modified Princeton profile monitor and is described in section 3. Following the detector is a Faraday cup for measuring the charge in each beam bunch. 


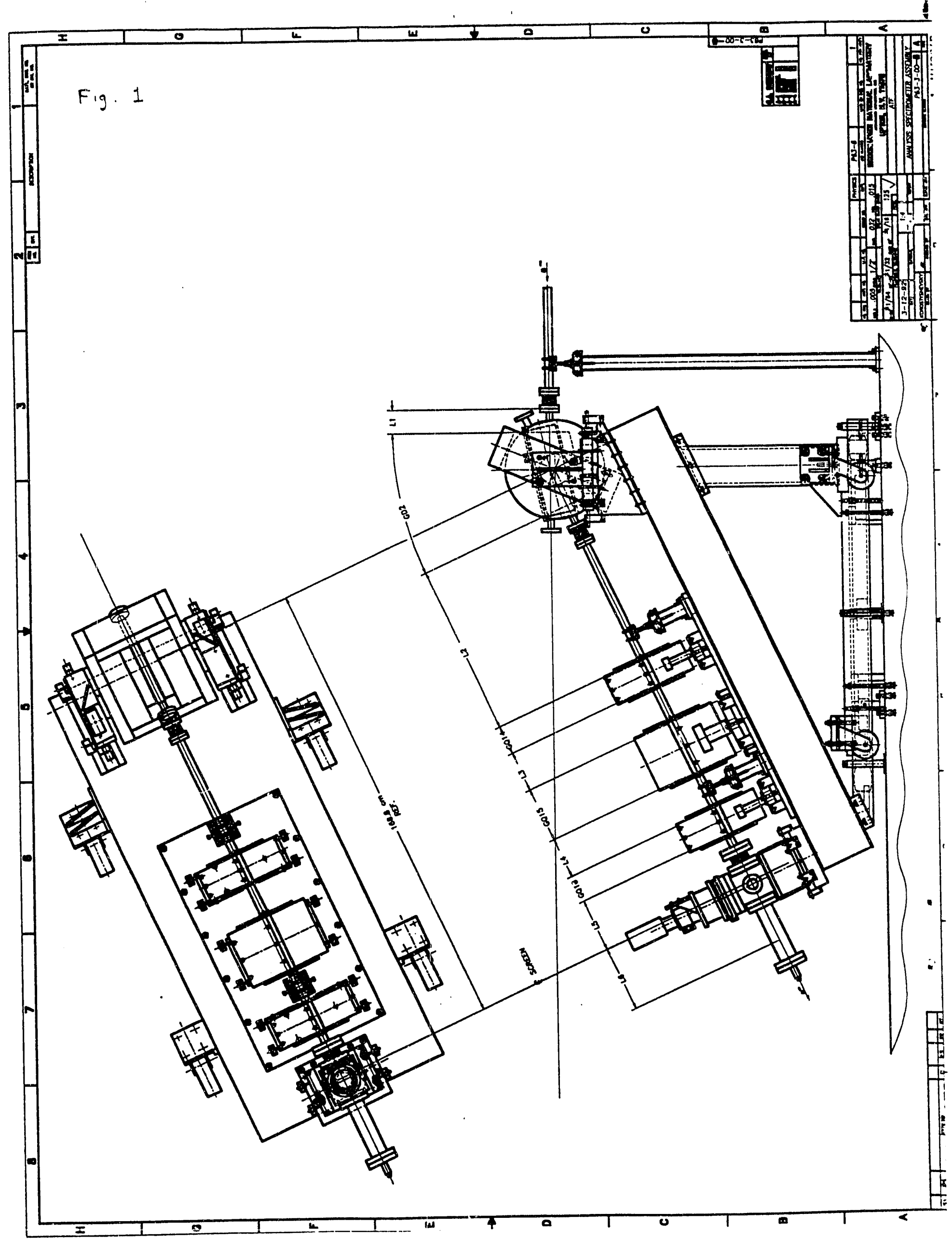


The initial set of acceleration experiments will not be able to control the phase of the interaction between the laser and the electrons. As a consequence the result of the interaction will be that some electrons will be accelerated, some decelerated, and others will be deflected. This means that the center of the spot produced on the detector will not move relative to a shot with the laser turned off, provided that the dipole is set to accept the mean incident beam momentum and that the experimental conditions are stable. Thus for these early measurements, where the total momentum change is no more than $\approx 1 \mathrm{MeV} / \mathrm{c}$, one is mainly interested in the width of the signal on the detector. The absolute location of the spot on the detector is determined by the mean beam momentum and is only of secondary importance for this measurement. All the essential information about the interaction comes from the size and shape of the spot itself, wherever the spot may lie on the detector plane. This is important because it means we can tolerate a certain amount of jitter from vibrations, magnet current variations, beam position drifts, etc. and not affect the spectrometer measurement.

When the experiments produce momentum gains larger than $\approx 1 \mathrm{MeV} / \mathrm{c}$, it will no longer be possible to see the entire distribution on the profile monitor at one time. In this case it will be necessary to use the spectrometer for an absolute measurement of momentum. The limits of the energy change distribution must be determined by changing the dipole field gradually until no further particles are seen on the profile monitor.

The strategy for the quadrupole settings is to have a point to point focus in the bending plane of the dipole. If we assume that the interaction region is small, all particles leaving with a given momentum are focused to a point on the detector, regardless of their initial divergence in that plane. However, particles with different momenta are focussed to different locations at the detector. Thus the location of the detector spot gives us information about the momentum change of the particles, but information about any deflections caused by the interaction in the bend plane will be lost. The quad tune in the non-bend plane should be roughly point to parallel, the main criterion being that the beam fall on the detector. The location of a trajectory at the detector in the non-bend direction should be proportional to the divergence in that direction caused by the interaction. Higher order effects in the magnetic optics will modify these statements to some extent. 
Some dimensional properties of the spectrometer are indicated in Table 1 .

\begin{tabular}{|l|c|c|c|}
\hline Table 1 & \multicolumn{3}{|c|}{ Spectrometer dimensions } \\
\hline el ement & length & Elength & $R_{\mathrm{p}}$ \\
\hline & $\mathrm{cm}$ & $\mathrm{cm}$ & $\mathrm{mm}$ \\
\hline L1 & 8.7 & 8.7 & \\
\hline GD2 & 28.2 & 36.9 & \\
\hline L2 & 63.9 & 100.8 & \\
\hline GQ14 & 10.2 & 111.0 & 15.2 \\
\hline L3 & 15.0 & 126.0 & \\
\hline GQ15 & 20.4 & 146.4 & 15.2 \\
\hline L4 & 15.0 & 161.4 & \\
\hline GQ16 & 10.2 & 171.6 & 15.2 \\
\hline L5 & 20.0 & 191.6 & \\
\hline L6 & 24.2 & 215.8 & \\
\hline
\end{tabular}

L 1 is the distance from the beam pipe flange to the dipole magnetic entrance edge. L5 is the distance from the last quad exit magnetic edge to the profile monitor screen. L6 is the distance from the screen to the entrance of the Faraday cup coilectior plate.

The beam pipe inner diameter between components in the spectrometer is $25.3 \mathrm{~mm}$. The smallest beam aperture is the entrance and exit beam pipes to the dipole vacuum chamber, where the inner diameter is $19.05 \mathrm{~mm}$.

\section{Magnetic measurements}

A circular coil dipole was used in the analys is spectrometer. The steel pole piece shown in Fig. 2 was designed for normal entrance and exit with a nominal bend angle of $25^{\circ}$. The edge of the pole was tapered such that the magnetic effective edge of the magnet would correspond with the physical edge. Although the measured angle between the entrance and exit pole edges was $24^{\circ} 48^{\prime}$, the entrance and exit beam pipes were surveyed at $25^{\circ}$. The gap between the dipole pole pieces is $3.099 \mathrm{~cm}$. The distance from the edge of the dipole pole piece to the theoretical bend point is $14.321 \mathrm{~cm}$. 


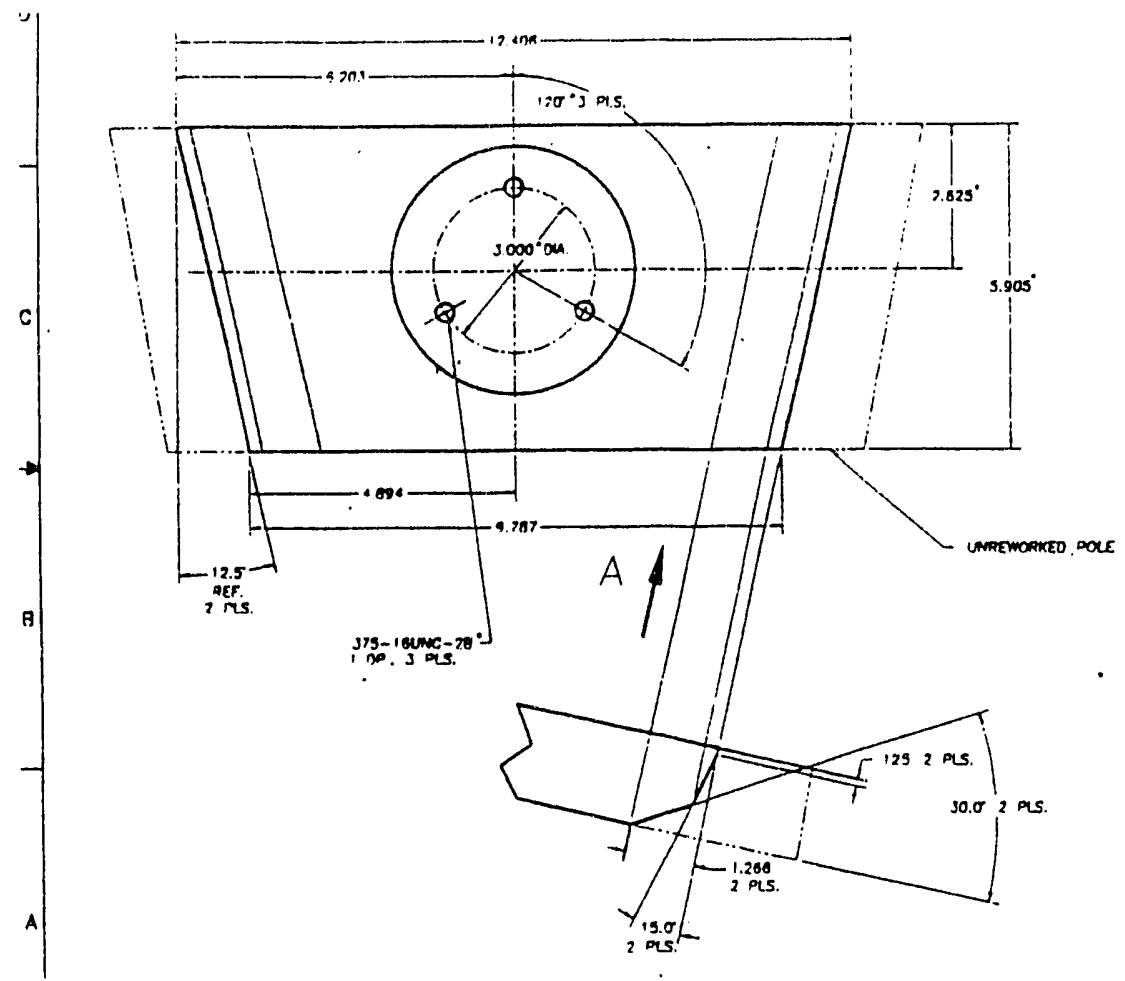

Fig. 2 Dipole pole piece.

The dipole was measured at the NSLS Magnetic Measurement lab. Hall probe measurements of the magnetic field along a line normal to the entrance face[5] are shown in Fig. 3. The probe entered the magnet at the center of the poleface width. These measurements confirmed that the magnetic edge of the magnet corresponded with the physical edge of the pole piece to within $1 \%$ of the total effective length.

\begin{tabular}{|c|c|c|}
\hline bend angle & 25 & degrees \\
\hline radius of curvature & 64.60 & $\mathrm{~cm}$ \\
\hline effective length & 28.19 & $\mathrm{~cm}$ \\
\hline sagitta & 1.53 & $\mathrm{~cm}$ \\
\hline$B$ for $50 \mathrm{MeV} / \mathrm{c}$ & 2.581 & $k G s$ \\
\hline
\end{tabular}

The excitation curve for the magnet is shown in Fig. 4. The Hall probe was located at the maximum field location[6] of Fig. 3. The field is linear with current up to about 2 kGs, after which saturation of the steel pole piece is clearly evident. 
Hiff jpectromeler - beom in adge

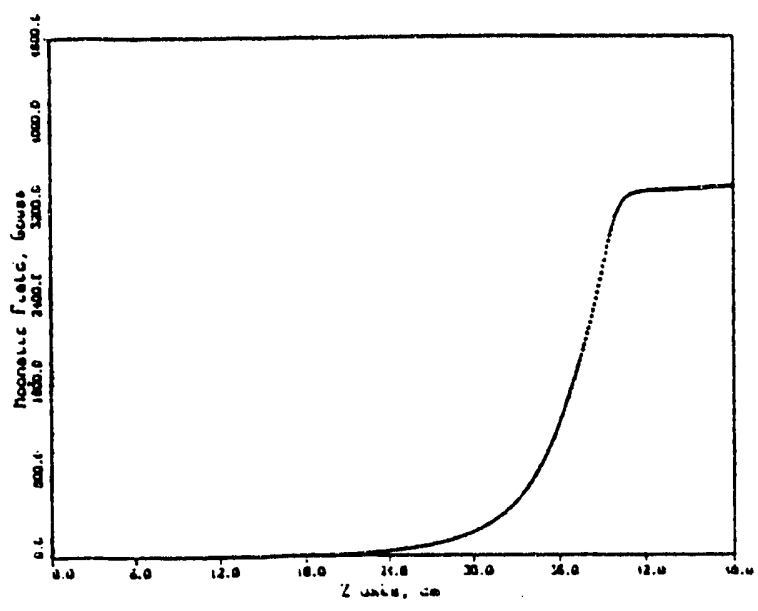

Fig. 3 Magnetic field in the dipole along trajectory perpendicular to the entrance face.

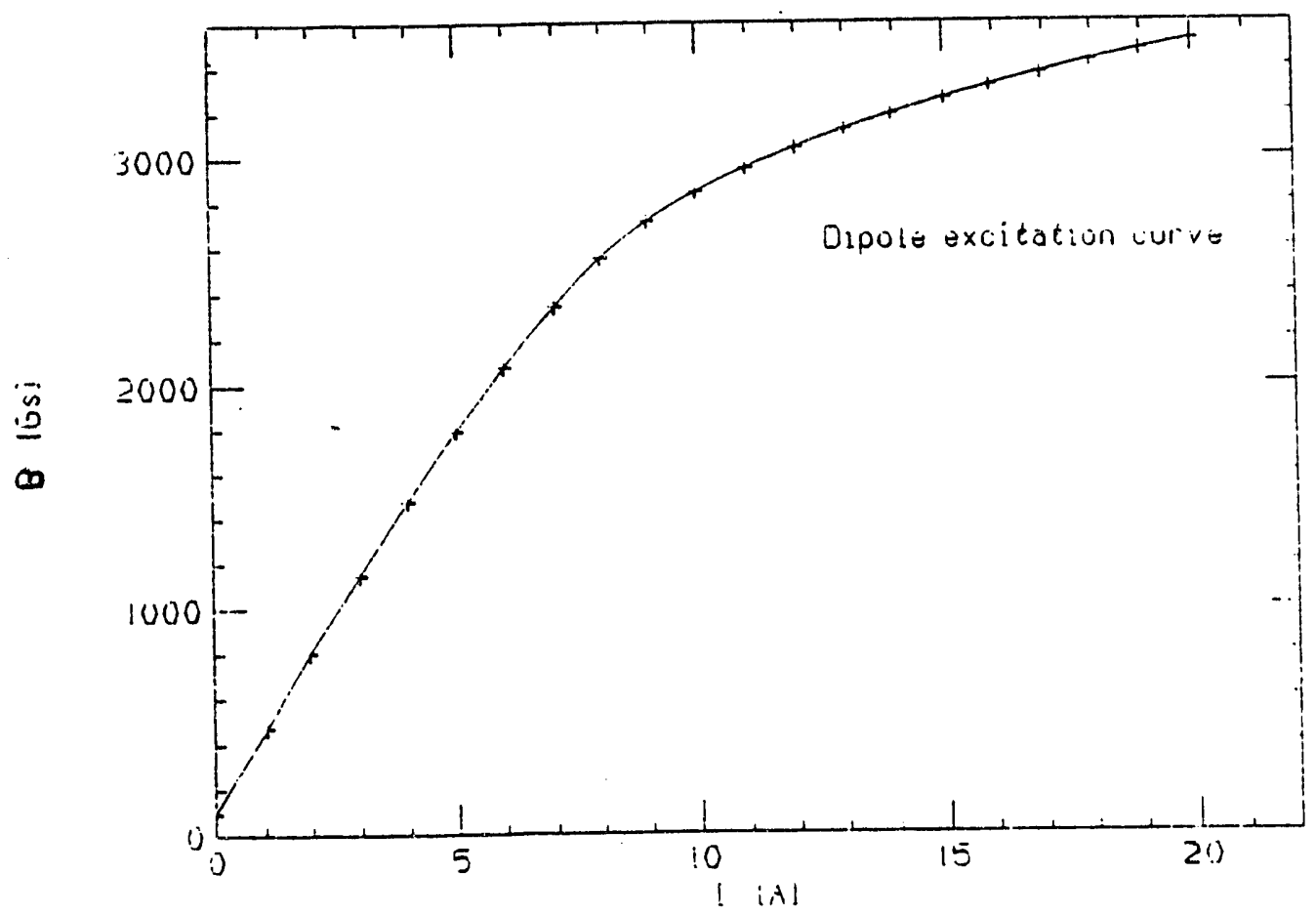

Fig. 4 Magnetic field in the dipole as a function of excitation current. 
3 Profile monitor assembly

The profile monitor is used to visually detect the electron beam at the exit of the spectrometer $[7,8]$. Fig. 5 shows a drawing of the profile monitor assembly. The beam first encounters an Al foil mounted perpendicularly to the beam axis. The exit side of the foil has $a \approx 15 \mathrm{~mm}$ diameter, layer of $G d_{,} O, S: T b$ phosphor. Scintillation light from the phosphor reflects from the $45^{\circ}$ mirror and passes through a vacuum window to the optical collection system. In the normal case this consists of a pair of lenses, f1 (Nikon) and f2 (Schneider), that make a point-to-point focus of a point on the phosphor to a point on the CCD camera (Pulnix model TM-745E). About $3 \%$ of the light is collected by the first lens and the transmission efficiency to the $C C D$ is $\approx 75 \%$. Approximately 4 charges are produced in a CCD pixel for each incident beam electron $[7,8]$.

The electron beam passes through the Al mirior on its way to the Faraday cup at the end of the beamline. A diagram of the Faraday cup is shown in Fig. 6. The mirror cannot be extracted like those in other ATF profile monitors, so the downstream side of the mirror was hollowed out. Table 3 shows a number of properties of the profile monitor system.

Table 3 Profile monitor properties

\begin{tabular}{|l|l|l|}
\hline \multicolumn{2}{|c|}{ Table 3 Profile monitor properties } \\
\hline phosphor thickness & 30 & $\mu \mathrm{m}$ \\
\hline phosphor density & 3 & $\mathrm{~g} / \mathrm{cm}^{3}$ \\
\hline phosphor grain size & 10 & $\mu \mathrm{m}$ \\
\hline phos 10\% decay time & 1.2 & $\mathrm{~ms}$ \\
\hline Al foil thickness & 25 & $\mu \mathrm{m}$ \\
\hline Al mirror thickness & 0.062 & $\mathrm{in}$ \\
\hline focal length f & 36 & $\mathrm{~mm}$ \\
\hline focal length $\mathrm{f}_{2}$ & 25.7 & $\mathrm{~mm}$ \\
\hline magnification & 0.71 & \\
\hline CCD active area & $8.4 \times 6.4$ & $\mathrm{~mm}$ \\
\hline spatial resolution & 50 & $\mu \mathrm{m}$ \\
\hline min observ beam inten & $2 \times 10^{6}$ & $\mathrm{~mm}$ \\
\hline
\end{tabular}




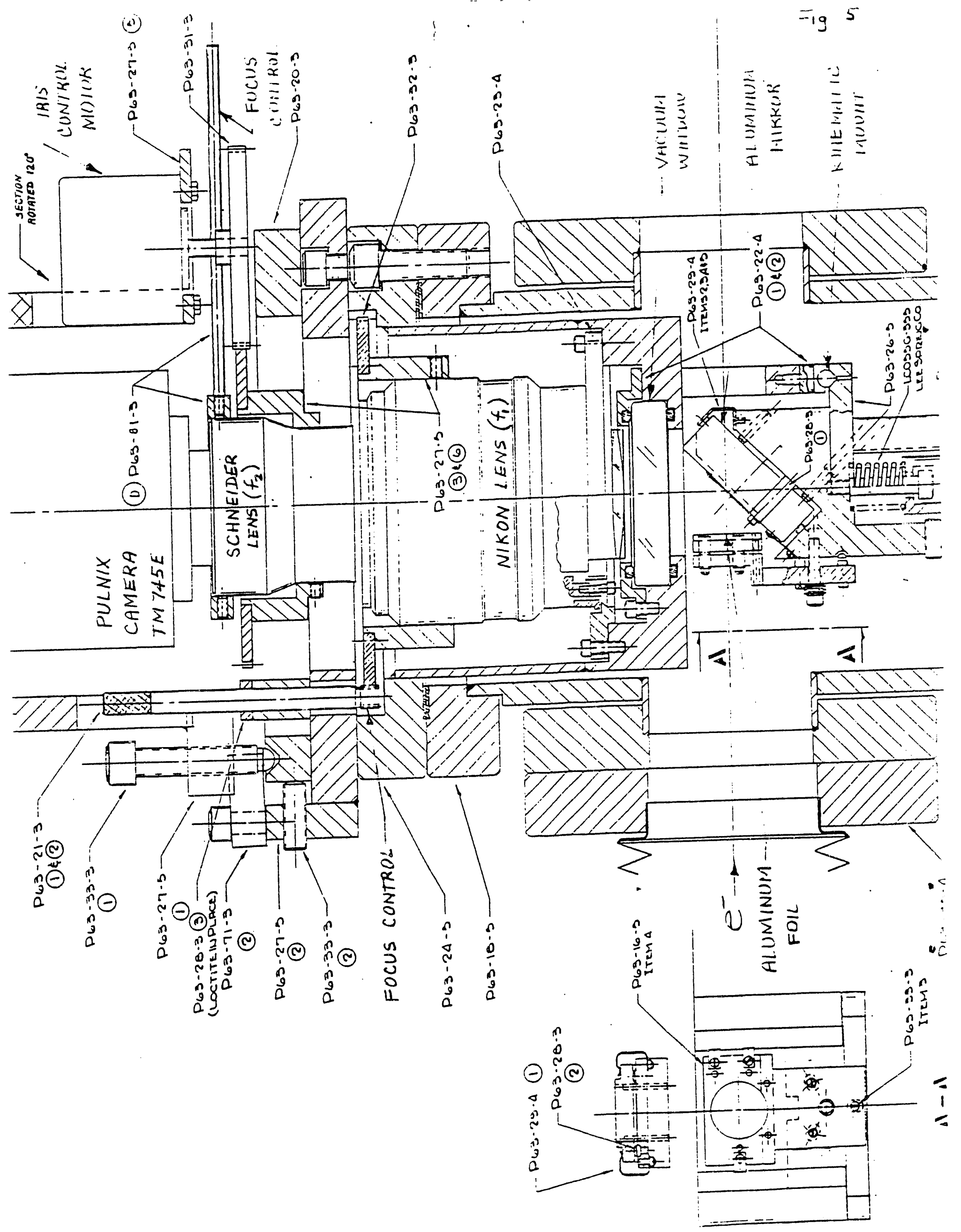




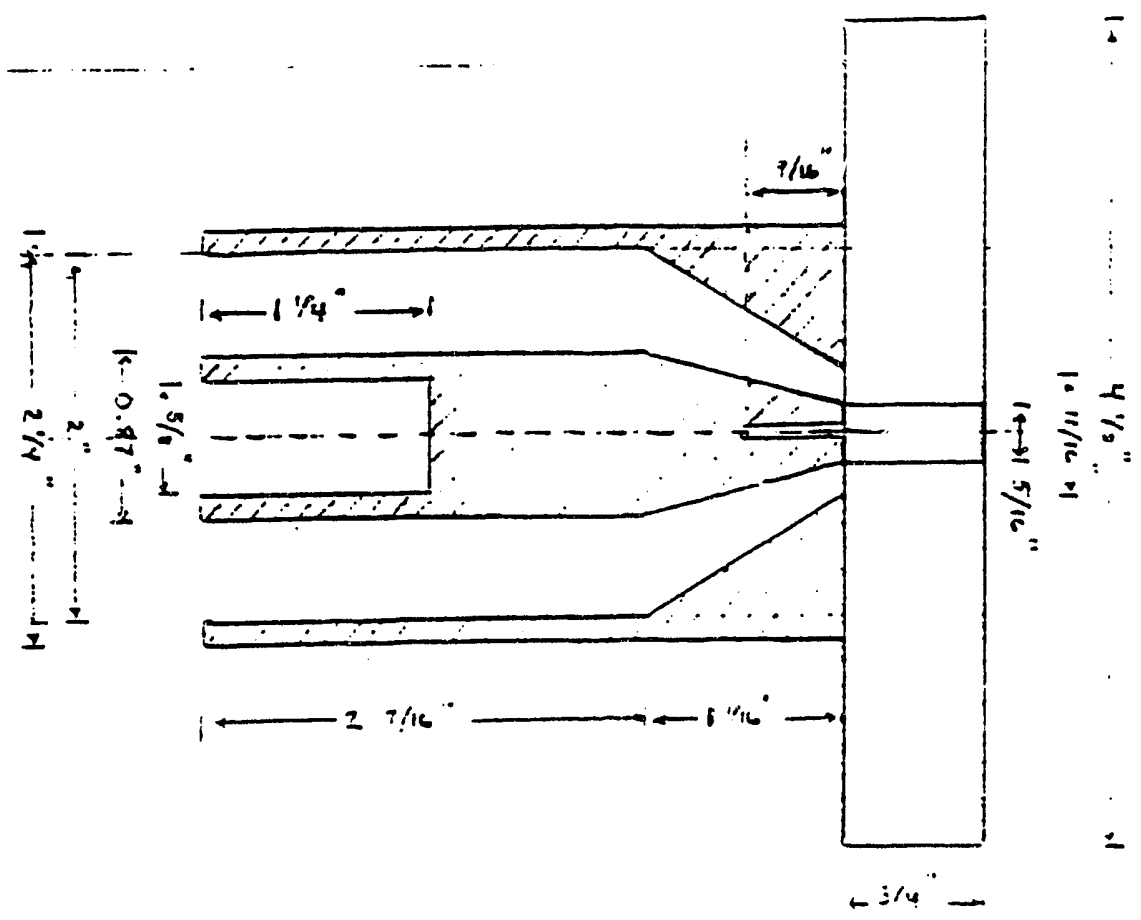

Fig. 6 Schematic diagram of the Princeton Faraday cup.

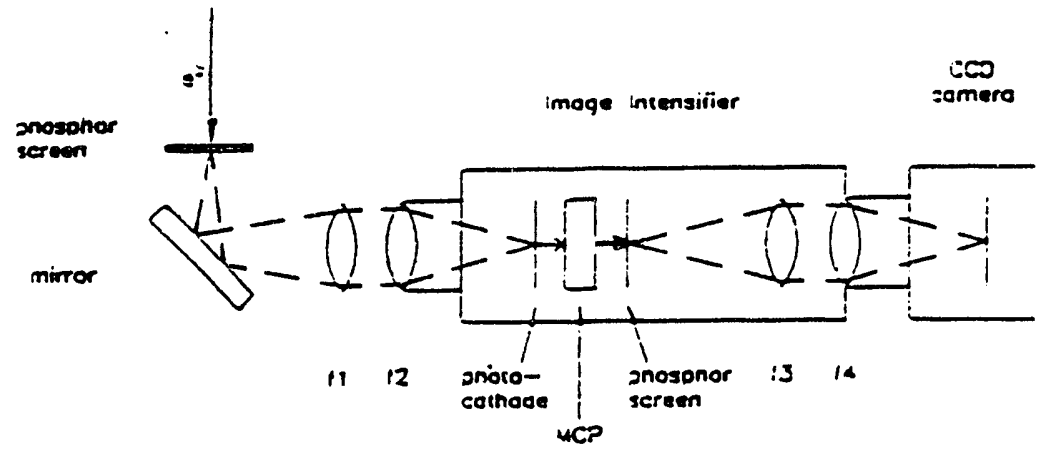

Fig. 7 Profile monitor optics with the image intensifier[?]. 
In situations where the beam spot is expected to be diffuse or where the total charge in the beam is small, an optional image intensifier can be used before the camera in the profile monitor. The intensifier, shown schematically in Fig. 7 and provided to the ATF by Princeton University, is a Paterson Electronics model LPQ50. It uses a $25 \mathrm{~mm}$, electrostatically focused microchannel plate. The gain is adjustable from 10000 to 50000. It is possible when using the intensifier to see single electrons with the profile monitor. The intensifier adds an additional contribution to the spatial resolution of $20 \mu \mathrm{m}$. This gives an overall spatial resolution of 55 $\mu \mathrm{m}$ when added in quadrature to the fundamental resolution of $50 \mu \mathrm{m}$ given in Table 3 . Lens $\mathrm{f} 3$ has a focal length of $50 \mathrm{~mm}$, while 44 has $35 \mathrm{~mm}$. Since this lens combination also has a magnificat ion of 0.7 , the overall magnification from the phosphor screen to the CCD camera is 0.50 .

\section{Expected performance}

We describe here typical values for the acceptances and resolution expected with this spectrometer. Some of these quantities are dependent on the actual geometrical layout of the experiment and the actual quadrupole settings.

\section{Angular acceptance}

We consider the non-bend plane acceptance for the case of the ICA experiment. The beampipe inner radius is $1.26 \mathrm{~cm}$. The first focussing quad in this plane is GQ15, whose entrance is located at a distance of $3.17 \mathrm{~m}$ from the center of the ICA gas cell. Thus the spectrometer should accept electrons leaving the ICA interaction region with deflections of up to $\pm 4.0 \mathrm{mR}$ in the non-bend plane[9].

\section{Momentum acceptance}

Consider an electron beam with mean momentum p. The change in bending angle $\alpha$ through dipole GD2 due to a change in momentum is given by

$$
\Delta \alpha=\alpha \frac{\Delta p}{p}
$$

This gives rise to a transverse displacement downstream of the dipole of

$$
\Delta \boldsymbol{x}=D \Delta \boldsymbol{\alpha}
$$

where $D$ is the distance from the center of the dipole to the center 
of the first bending plane focussing quad GQ14. The momentum range accepted by the spectrometer is

$$
\Delta p=\frac{\Delta x}{\alpha D} p
$$

Using $\Delta x=1.26 \mathrm{~cm}$ for the beampipe inner radius, $D=83.2 \mathrm{~cm}$, and a central momentum $p=45 \mathrm{MeV} / \mathrm{c}$, we find that the momentum range accepted by the spectrometer is $\pm 1.6 \mathrm{MeV} / \mathrm{c}$.

For an optimal design the momentum measurement range on the profile monitor screen should match the momentum acceptance above. The transverse displacement caused by momentum spread is given by the $\mathrm{R}_{16}$ matrix element in TRANSPORT. The nominal spectrometer tune has

$$
\begin{aligned}
R_{16} & =2.0 \frac{\mathrm{mm}}{8} \\
& =4.4 \frac{\mathrm{mm}}{\mathrm{MeV} / \mathrm{C}}
\end{aligned}
$$

where the second 1 ine applies to a central momentum of $45 \mathrm{MeV} / \mathrm{c}$. Using a screen half-width of $7.5 \mathrm{~mm}$, this gives a measured momentum range $\pm 1.7 \mathrm{MeV} / \mathrm{c}$.

\section{Momentum resolution}

We define the momentum resolution to be the smallest measurable momentum change. Taking $50 \mu \mathrm{m}$ to be the spatial resolution of the profile monitor, and using the nominal $R_{16}=4.4$ given above, the momentum resolution of the spectrometer is $12 \mathrm{keV} / \mathrm{c}$.

\section{Beam profile}

We give an example beam profile through the spectrometer in Fig. 8 . The figure shows the 1 sigma beam envelope in the bend and non-bend planes versus axial distance $Z$ for the ICA experiment. $Z=0$ corresponds to the exit window of the ICA gas cell. The curves extend to $Z \approx 360 \mathrm{~cm}$, which corresponds to the screen in the profile monitor detector. The quadrupoles produce point-to-point focussing in the bend plane (the starting beam size is not actually a point because of multiple scattering in the gas and windows). 


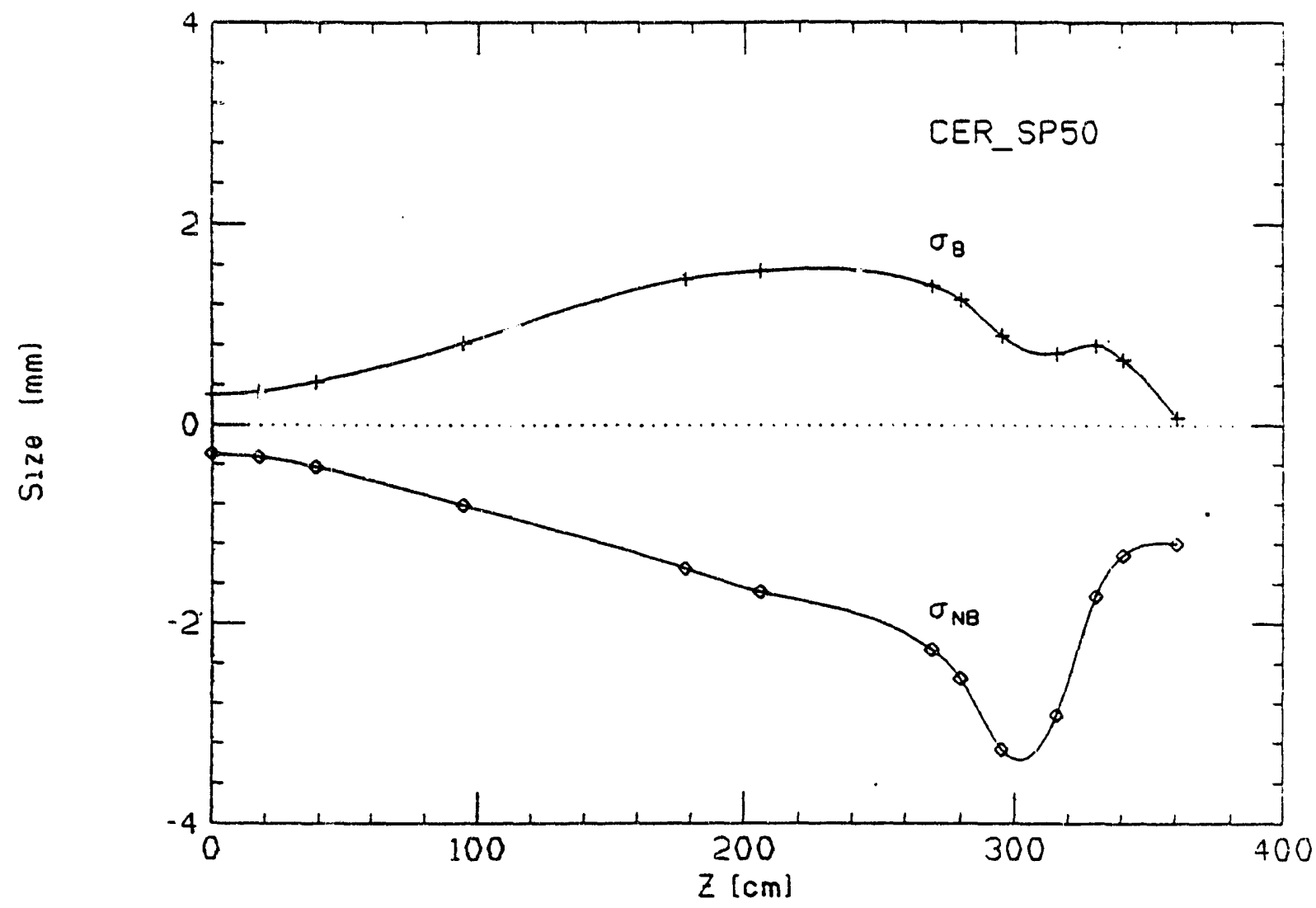

Fig. 8 Beam profiles in the bend (B) and non-bend (NB) planes in the analysis spectrometer for the ICA expariment.

\section{Acknow ledgements}

We would like to thank Kirk McDonald, Pat Russell, Lorraine Solomon, and $\mathrm{Xijie}$ wang for their assistance in this work. 


\section{Notes and References}

[1] W. Kimura et al, Inverse Cerenkov laser acceleration experiment, Spectra Technology Inc. note DE-AC06-83ER40128, 1989. [2] W. Chen et al, Proposal for a study of laser acceleration of electrons using micrograting structures at the ATF, Brookhaven National Laboratory report, BNL-43465, 1989.

[3] K.T. McDonald et al, Proposal for an experimental study of nonlinear Compton scattering, Princeton University note DOE/ER/3072-55, 1989.

[4] The quadrupoles were located in front of the dipole in the original Grating Acceleration experiment designs. See for example, R.C. Fernow, Updated $50 \mathrm{MeV}$ spectrometer design, informal report RCF-LA-8602, 1986; R.C. Fernow, scintillating fiber detector for the grating acceleration spectrometer, informal report RCF-LA-8801, 1988. This arrangement would have allowed a range of momentum acceptances to be covered by adjusting the distance between the dipole and the profile monitor. The design was changed in 1989 with the quads moved downstream of the dipole to accommodate simultaneous running of the Grating Acceleration and Compton scattering experiments.

[5] Note that this measurement path crosses the exit edge at an angle of $25^{\circ}$.

[6] The location of the true maximum field was measured to be $13 \mathrm{~mm}$ transverse to this point, where the field was 1.2 Gs higher.

[7] D.P. Russell II I, High-brightness electron beam production at the Brookhaven Accelerator Test Facility, Dissertation, Princeton University, 1992; DOE/ER/3072-67. Chapter 7 describes the profile monitor system, including the image intensifier.

[8] D.P. Russell \& K.T. McDonald, A beam profile monitor for the BNL Accelerator Test Facility, 1989 Part. Acc. Conf.; DOE/ER/307252 .

[9] This ignores the defocussing effect of the preceding quad GQ14. The extra divergence is negligible for the standard ICA beam tune.

\section{Appendix: magnet excitation curves}

For completeness we include here excitation curves for each type of magnet used in the spectrometer. The short and long SLAC quads following the dipole are integral parts of the spectrometer assembly. The long Chinese quads, located upstream of the dipole, can optionally be used for some experimental arrangements. The quadrupole excitation curves are taken from $X$. Wang \& $C$. Sylvester, Quadrupole magnets measurements, informal report, BNL-45801. 


\begin{tabular}{|c|c|c|}
\hline Table A1 & ion cur & GD2 dipole \\
\hline$I \quad[A]$ & $B$ [Gs] & $p[\mathrm{MeV} / \mathrm{C}]$ \\
\hline 0.000 & 95.6 & 1.85 \\
\hline 1.034 & 471.3 & 9.13 \\
\hline 1.964 & 802.5 & 15.55 \\
\hline 2.986 & 1149.0 & 22.26 \\
\hline 3.985 & 1477.7 & 28.63 \\
\hline 4.995 & 1789.6 & 34.68 \\
\hline 5.988 & 2077.0 & 40.24 \\
\hline 7.002 & 2343.0 & 45.40 \\
\hline 7.973 & 2553.3 & 49.47 \\
\hline 8.971 & 2716.9 & 52.64 \\
\hline 9.980 & 2845.0 & 55.13 \\
\hline 10.977 & 2949.6 & 57.15 \\
\hline 11.998 & 3041.9 & 58.94 \\
\hline 13.026 & 3123.7 & 60.53 \\
\hline 13.962 & 3190.8 & 61.83 \\
\hline 15.016 & 3259.4 & 63.15 \\
\hline 15.931 & 3314.0 & 64.21 \\
\hline 16.950 & 3370.4 & 65.31 \\
\hline 17.992 & 3423.7 & 66.34 \\
\hline 18.996 & 3471.0 & 67.25 \\
\hline 20.000 & 3514.7 & 68.10 \\
\hline
\end{tabular}




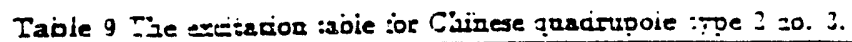

\begin{tabular}{|c|c|}
\hline Currens $(A)$ & Gradiens! Gauss / $\simeq$ I \\
\hline 3.5 & .9 .22295 \\
\hline$\therefore .0$ & $: 39.327573$ \\
\hline$\vdots 0.5$ & 238.0729 6ta \\
\hline$\therefore \div 0$ & 317.5825 \\
\hline$\therefore .5$ & 395.99225 \\
\hline $2 \pm .1$ & $\div 5.09525$ \\
\hline 24.5 & 533.531 \\
\hline
\end{tabular}

Table 10 The exciacion :abie :or sior: SLiC quacirspoie t53.

\begin{tabular}{|c|c|c|c|}
\hline Cucrenr( $(-1)$ & Gradiens (C & & \\
\hline$\overline{0.7}$ & 236.3408 & & $1.36 \mathrm{~T} / \mathrm{m}$ \\
\hline$i . \div$ & 252.5708, & & \\
\hline 2.1 & 377.4048 & & \\
\hline 2.8 & 493.424 & & \\
\hline 3.2 & 512.8535 & & \\
\hline 42 & 731.4085 & & \\
\hline 4.9 & 849.028 & & \\
\hline 5.6 & $\begin{array}{l}964.3245 \\
\ldots\end{array}$ & & \\
\hline 5.2 & 1080.4165 & & \\
\hline$: .0$ & 1186.6385 . & & \\
\hline
\end{tabular}

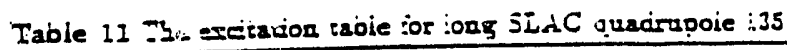

\begin{tabular}{|c|c|}
\hline Cursend $(i)$ & Gradiensi Gauss/ $=$ i \\
\hline 0.7 & $: 11.34233$ \\
\hline$\therefore .1$ & $25 \pi .78066,: 32.1486$ \\
\hline $2 \therefore$ & $2 \pi 7.929333$ \\
\hline 2.3 & $\div 99.5900$ \\
\hline $3 . \Sigma$ & 521.2633 \\
\hline 12 & $i 43.160667$ \\
\hline$\therefore 2$ & $\$ 66.03723$. \\
\hline j.0 & $388.51: 60 \%$ \\
\hline$j .2$ & $:=09 . \div 165$ \\
\hline$\therefore$ & $: \because 25.52866$ \\
\hline
\end{tabular}



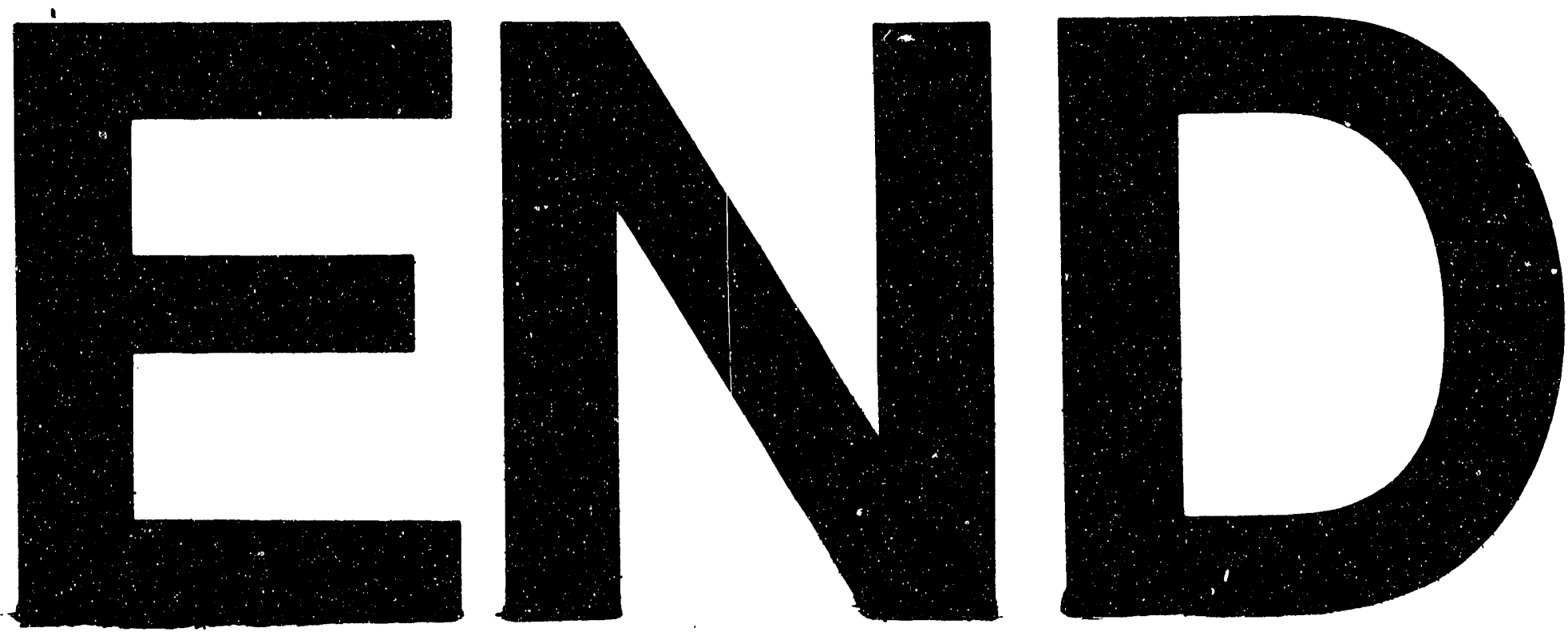

$x$
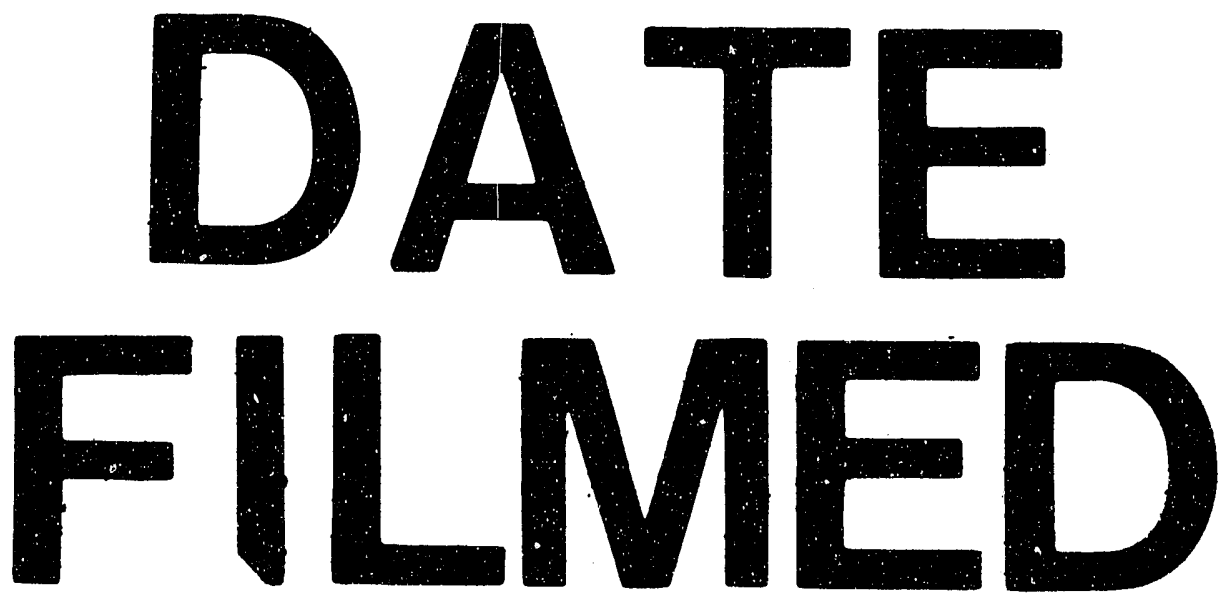

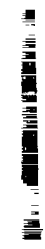

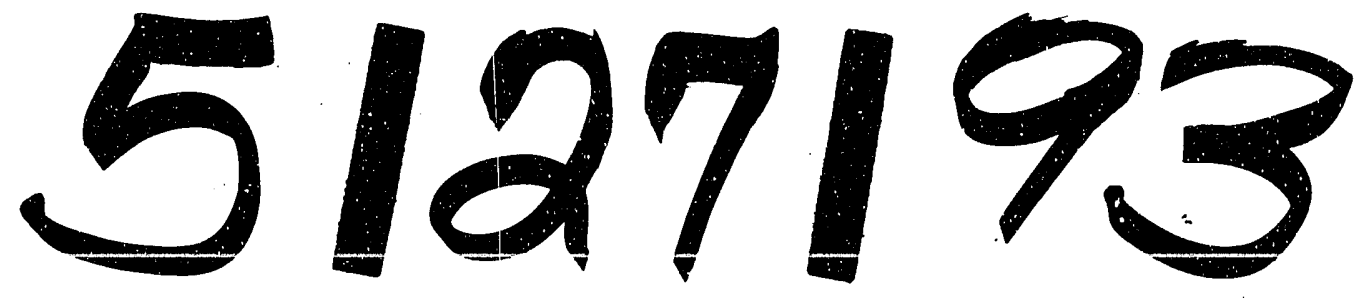


